\title{
NONEXISTENCE OF POSITIVE SOLUTIONS OF A NONLINEAR PARTIAL DIFFERENCE EQUATION
}

\author{
SHU TANG LIU AND SUI SUN CHENG
}

\begin{abstract}
Necessary conditions are derived for the existence of positive solutions of a class of nonlinear partial difference equations. The technique used to derive these conditions is based on the nonexistence of positive roots of an associated characteristic equation.
\end{abstract}

\section{Introduction} form

This paper is concerned with a class of nonlinear partial difference equations of the

$$
\left(u_{m+1, n}+u_{m, n+1}\right)^{2}-u_{m n}^{2}+\sum_{i=1}^{k} p_{i}(m, n) u_{m-\sigma_{i}, n-\tau_{i}}^{2}=0
$$

defined for $m, n=0,1,2, \cdots$, where $p_{1}(m, n), \cdots, p_{k}(m, n)$ are positive functions defined for $m, n \geq 0$, and the "delays" $\sigma_{1}, \cdots, \sigma_{k}, \tau_{1}, \cdots, \tau_{k}$ are nonnegative integers. Since (1) can be written as

$$
u_{m, n+1}= \pm \sqrt{u_{m n}^{2}-\sum_{i=1}^{k} p_{i}(m, n) u_{m-\sigma_{i}, m-\tau_{i}}^{2}}-u_{m+1, n}
$$

it is clear that given initial conditions $u_{m n}=\phi_{m n}$ for each $(m, n)$ in

$$
\{(m, n) \mid m \geq-\sigma, n \geq-\tau\} \backslash\{(m, n) \mid m \geq 0, n \geq 1\}
$$

where

$$
\sigma=\max \left\{\sigma_{1}, \cdots, \sigma_{k}\right\}, \text { and } \tau=\max \left\{\tau_{1}, \cdots, \tau_{k}\right\},
$$

we may calculate a double sequence $u_{01} ; u_{11}, u_{02} ; u_{21}, u_{12}, u_{03} ; u_{31}, u_{22}, u_{13}, u_{04}$; ... successively. This double sequence may be complex valued and its terms depend on the sign of a square root which we choose at each step of computation. Nevertheless, a double sequence $u=\left\{u_{m n}\right\}$ defined for $m \geq-\sigma$ and $n \geq-\tau$ will be called a solution of

Received April 19, 1996.

1991 Mathematics Subject Classification. Primary 39A10.

Key words and phrases. Nonlinear partial difference equations, characteristic equations, positive solutions. 
(1) if (1) is satisfied by $u$. Here we are concerned with the question as to when (1) does not have an eventually positive solution $u=\left\{u_{m n}\right\}$, i.e. $u_{m n}>0$ for all large $m$ and $n$.

This study is motivated by $[2,3]$ in which linear partial difference inequalities of the form

$$
v_{m+1, n}+v_{m, n+1}-v_{m n}+p(m, n) v_{m-\sigma, n-\tau} \leq 0,
$$

is investigated. An immediate connection between our nonlinear equation (1) and (2) is clear from rewriting (1) as

$$
u_{m+1, n}^{2}+u_{m, n+1}^{2}-u_{m n}^{2}+\sum_{i=1}^{k} p_{i}(m, n) u_{m-\sigma_{i}, n-\tau_{i}}^{2}=-2 u_{m+1, n} u_{m, n+1} .
$$

Therefore, if $u=\left\{u_{m n}\right\}$ is an eventually positive solution of (3), then $v=\left\{u_{m n}^{2}\right\}$ will be an eventually positive solution of (2).

We remark further that equation (1) may also be regarded as a discrete analog of partial differential equations of the form

$$
\left(u_{x}+u_{y}+2 u\right)^{2}-u^{2}+\sum_{i=1}^{k} p_{i}(x, y) u^{2}\left(x-\sigma_{i}, y-\tau_{i}\right)=0 .
$$

Therefore, qualitative properties of (1) may yield useful information for this companion partial differential equation.

We first give an example to show that eventually positive solutions may exist under appropriate conditions.

Example 1. Assume either one of the nonnegative integers $\sigma$ and $\tau$ is not zero, that $p>0$ and that

$$
\left(4^{\sigma+\tau}(\sigma+\tau) p\right)^{1 /(\sigma+\tau+1)} \frac{\sigma+\tau+1}{\sigma+\tau} \leq 1 .
$$

Then the equation

$$
\left(u_{m+1, n}+u_{m, n+1}\right)^{2}-u_{m n}^{2}+p u_{m-\sigma, n-\tau}^{2}=0, m, n \geq 0 .
$$

has an eventually positive solution.

Indeed, we look for a positive solution of the form

$$
u_{m n}=\lambda^{m+n}
$$

Substituting this function into (5), we obtain

$$
\Gamma(\lambda) \equiv 4 \lambda^{2}-1+p \lambda^{-2(\sigma+\tau)}=0 .
$$

Note that $\Gamma(+\infty)=+\infty$ and

$$
\min _{\lambda>0} \Gamma(\lambda)=\left(4^{\sigma+\tau}(\sigma+\tau) p\right)^{1 /(\sigma+\tau+1)} \frac{\sigma+\tau+1}{\sigma+\tau}-1 \leq 0 .
$$


Thus, $\Gamma(\lambda)$ has a positive root, which shows that our guess is valid.

Although general conditions for the existence of eventually positive solutions of (1) are not clear at this point, however, we are able to find several sets of necessary conditions in the next section.

In the sequel, we will adopt the convention that an empty product equals one.

\section{Nonexistence Criteria}

As we have remarked before, if $u=\left\{u_{m n}\right\}$ is an eventually positive solution of (3), then $v=\left\{u_{m n}^{2}\right\}$ will be an eventually positive solution of (2). Thus necessary conditions for the existence of eventually positive solutions of (2) are also necessary conditions for (3). We will therefore pay attention to nonexistence criteria which are different from those obtained in $[2,3]$. We first note the following decreasing nature of an eventually positive solution of (1).

Lemma 1. An eventually positive solution $u=\left\{u_{m n}\right\}$ of (1) is also eventually decreasing with respect to both of its independent variables.

This follows from

$$
\begin{aligned}
& \max \left\{u_{m+1, n}^{2}-u_{m n}^{2}, u_{m, n+1}^{2}-u_{m n}^{2}\right\} \\
\leq & u_{m+1, n}^{2}+u_{m, n+1}^{2}-u_{m n}^{2}+\sum_{i=1}^{k} p_{i}(m, n) u_{m-\sigma_{i}, n-\tau_{i}}^{2} \\
= & -2 u_{m+1, n} u_{m, n+1}<0
\end{aligned}
$$

for all large $m$ and $n$.

For the sake of convenience, we will set

$$
\liminf _{m, n \rightarrow \infty} p_{i}(m, n)=p_{i}, 1 \leq i \leq k,
$$

and

$$
\limsup _{m, n \rightarrow \infty} p_{i}(m, n)=P_{i}, l \leq i \leq k .
$$

Theorem 1. Assume that $\sigma_{i}, \tau_{i} \geq 1$ and that $p_{i}>0$ for $1 \leq i \leq k$. If (1) has an eventually positive solution, then

$$
4 \sum_{i=1}^{k} p_{i}+\sum_{i=1}^{k} P_{i} \leq 1 .
$$

Proof. Assume that $u=\left\{u_{m n}\right\}$ is an eventually positive solution of (1). Let $\epsilon$ be an arbitrary number which satisfies $0<\epsilon<\min \left\{p_{i}, \cdots, p_{k}\right\}$. Then there exist integers $M$ 
and $N$ such that $u_{n m}>0$ and $p_{i}(m, n) \geq p_{i}-\epsilon$ for $m \geq M, n \geq N$ and $1 \leq i \leq k$. In view of (1), we have

$$
\sum_{i=1}^{k} p_{i}(m, n) u_{m-\sigma_{i}, n-\tau_{i}}^{2}<u_{m n}^{2} .
$$

Thus by means of the decreasing nature of $u$, and by means of $\sigma_{i}, \tau_{i} \geq 1$ for $1 \leq i \leq k$, we see that

$$
u_{m n}^{2}>\sum_{i=1}^{k} p_{i}(m, n) u_{m-1, n-1}^{2}>\sum_{i=1}^{k}\left(p_{i}-\epsilon\right) u_{m-1, n}^{2}
$$

for all large $m$ and $n$. Similarly, we have

$$
u_{m n}^{2}>\sum_{i=1}^{k}\left(p_{i}-\epsilon\right) u_{m, n-1}^{2}
$$

for all large $m$ and $n$. In view of (1) again, we now see that

$$
\begin{aligned}
0 & =\left(u_{m+1, n}+u_{m, n+1}\right)^{2}-u_{m n}^{2}+\sum_{i=1}^{k} p_{i}(m, n) u_{m-\sigma_{i}, m-\tau_{i}}^{2} \\
& >4 \sum_{i=1}^{k}\left(p_{i}-\epsilon\right) u_{m n}^{2}-u_{m n}^{2}+\sum_{i=1}^{k} p_{i}(m, n) u_{m n}^{2} \\
& =u_{m n}^{2}\left\{4 \sum_{i=1}^{k}\left(p_{i}-\epsilon\right)-1+\sum_{i=1}^{k} p_{i}(m, n)\right\}
\end{aligned}
$$

which implies

$$
4 \sum_{i=1}^{k}\left(p_{i}-\epsilon\right)+\sum_{i=1}^{k} p_{i}(m, n)<1
$$

By taking the superior limits on both sides of the above inequality as $m, n$ tend to infinity, and then the limits as $\epsilon$ tends to zero, we will arrive at (6). The proof is complete.

As an example, consider the equation

$$
\left(u_{m+1, n}+u_{m, n+1}\right)^{2}-u_{m n}^{2}+\frac{\left(m^{2}+2 m\right)(m-2)^{2}}{m^{2}(m+1)^{2}} u_{m-2, n-1}^{2}=0
$$

Since

$$
\lim _{m, n \rightarrow \infty} \frac{\left(m^{2}+2 m\right)(m-2)^{2}}{m^{2}(m+1)^{2}}=1,
$$

condition (6) cannot hold. Thus (7) cannot have any eventually positive solutions. It is interesting to note that $u=\left\{(-1)^{n} / m\right\}$ is an "oscillating" solution of (7).

The proof of Theorem 1 will not work if $\sigma=0$ or $\tau=0$. In case $\sigma=0$ or $\tau=0$ but one of $\sigma$ or $\tau$ is not zero, we have the following result. 
Theorem 2. Assume that $\max \left\{\sigma_{1}, \cdots, \sigma_{k}, \tau_{1}, \cdots, \tau_{k}\right\} \geq 1$. If (1) has an eventually positive solution, then

$$
\sum_{i=1}^{k} p_{i} 4^{\gamma_{i}} \frac{\left(\gamma_{i}+1\right)^{\gamma_{i}+1}}{\gamma_{i}^{\gamma_{i}}} \leq 1
$$

where $\gamma_{i}=\min \left\{\sigma_{i}, \tau_{i}\right\}$ for $i=1,2, \cdots, k$.

Proof. Assume that $u=\left\{u_{m n}\right\}$ is an eventually positive solution of (1). By (1) and the decreasing nature of $u$, we see that

$$
\begin{aligned}
& \left(\frac{2 u_{m+1, n+1}}{u_{m n}}\right)^{2}-1 \leq\left(\frac{u_{m+1, n}+u_{m, n+1}}{u_{m n}}\right)^{2}-1 \\
= & -\sum_{i=1}^{k} p_{i}(m, n) \frac{u_{m-\sigma_{i}, n-\tau_{i}}^{2}}{u_{m n}^{2}} \leq-\sum_{i=1}^{k} p_{i}(m, n) \frac{u_{m-\gamma_{i}, m-\gamma_{i}}^{2}}{u_{m n}^{2}} .
\end{aligned}
$$

Note that

$$
\frac{u_{m-\gamma_{i}, n-\gamma_{i}}}{u_{m n}}=\frac{u_{m-\gamma_{i}, n-\gamma_{i}}}{u_{m-\gamma_{i}+1, n-\gamma_{i}+1}} \frac{u_{m-\gamma_{i}+1, m-\gamma_{i}+1}}{u_{m-\gamma_{i}+2, m-\gamma_{i}+2}} \cdots \frac{u_{m-1, m-1}}{u_{m n}}
$$

thus, by letting

$$
w_{m n}=\frac{u_{m n}}{u_{n+1, n+1}}
$$

we see that $w_{m n}>1$ and

$$
\left(\frac{2}{w_{m n}}\right)^{2}+\sum_{i=1}^{k} p_{i}(m, n)\left(\prod_{j=1}^{\gamma_{i}} w_{m-j, n-j}\right)^{2} \leq 1
$$

for all large $m$ and $n$. Therefore, $\left\{w_{m n}\right\}$ is bounded in view of the assumption that $\max \left\{\sigma_{1}, \cdots, \sigma_{k}, \tau_{1}, \cdots, \tau_{k}\right\} \geq 1$. Furthermore, letting

$$
w=\liminf _{m, n \rightarrow \infty} w_{m n}
$$

we see that $w \geq 1$, and from (9) that

$$
\begin{aligned}
\left(\frac{2}{w}\right)^{2} & =\limsup _{m, n \rightarrow \infty}\left(\frac{2}{w_{m n}}\right)^{2} \\
& \leq 1-\sum_{i=1}^{k} \liminf _{m, n \rightarrow \infty}\left\{p_{i}(m, n)\left(\prod_{j=1}^{\gamma_{i}} w_{m-j, n-j}\right)^{2}\right\} \\
& \leq 1-\sum_{i=1}^{k} p_{i} w^{2 \gamma_{i}} .
\end{aligned}
$$

Therefore, $w$ cannot belong to the close interval $[1,2]$, and we may then rewrite the above inequality as

$$
\Phi(w) \equiv \sum_{i=1}^{k} \frac{p_{i} w^{2 \gamma_{i}+2}}{w^{2}-4} \leq 1, w>2 .
$$


Note that

thus

$$
\min _{w>2} \frac{w^{2 \gamma_{i}+2}}{w^{2}-4}=\frac{4^{\gamma_{i}}\left(\gamma_{i}+1\right)^{\gamma_{i}+1}}{\gamma_{i}^{\gamma_{i}}}
$$

$$
\min _{w>2} \Phi(w)=\sum_{i=1}^{k} p_{i} \frac{4^{\gamma_{i}}\left(\gamma_{i}+1\right)^{\gamma_{i}+1}}{\gamma_{i}^{\gamma_{i}}} \leq 1
$$

The proof is complete.

As an example, consider the equation

$$
\left(u_{m+1, n}+u_{m, n+1}\right)^{2}-u_{m n}^{2}+\frac{n^{2}-1}{(n-2)^{2}} u_{m-2, n-1}^{2}=0 .
$$

Here, $\sigma=1, \tau=2$ and

$$
\lim _{m, n \rightarrow \infty} \frac{n^{2}-1}{(n-2)^{2}}=1
$$

Therefore, the left hand side of (8) is 16 , which is greater than 1 . We may now conclude that (11) does not have any eventually positive solutions. It is interesting to note that $\left\{(-1)^{m} n\right\}$ is an "oscillating" solution of (11).

There are two variants of the above Theorem. The first one is a 1-norm type inequality condition [1] for the existence of an eventually positive solution of (1).

Theorem 3. Suppose $\eta=\min \left\{\sigma_{1}, \cdots, \sigma_{k}, \tau_{1}, \cdots, \tau_{k}\right\} \geq 1$. If (1) has an eventually positive solution, then

$$
\sum_{i=1}^{k} p_{i} \leq\left(\frac{\eta}{4}\right)^{\eta} \frac{1}{(\eta+1)^{\eta+1}}
$$

As seen in the proof of Theorem 2, if $u=\left\{u_{m n}\right\}$ is an eventually positive solution of (1), then the lower limit $w$ defined by (10) will satisfy $w>2$ and

$$
\left(\frac{2}{w}\right)^{2} \leq 1-\sum_{i=1}^{k} p_{i} w^{2 \gamma_{i}} \leq 1-w^{2 \eta} \sum_{i=1}^{k} p_{i}
$$

Thus

$$
\sum_{i=1}^{k} p_{i} \leq \frac{w^{2}-4}{w^{2(\eta+1)}} \leq \max _{w>2} \frac{w^{2}-4}{w^{2(\eta+1)}}=\left(\frac{\eta}{4}\right)^{\eta} \frac{1}{(\eta+1)^{\eta+1}}
$$

as required.

We remark that in the above Theorem, the condition $\eta \geq 1$ is used in deriving the maximum of $\left(w^{2}-4\right) / w^{2(\eta+1)}$ over $(w, \infty)$.

Theorem 4. Assume that $\max \left\{\sigma_{1}, \cdots, \sigma_{k}, \tau_{1}, \cdots, \tau_{k}\right\} \geq 1$. If (1) has an eventually positive solution, then

$$
k\left(\prod_{i=1}^{k} p_{i}\right)^{1 / k} \leq \frac{8}{2^{\zeta \zeta}}\left(\frac{\zeta-2}{\zeta}\right)^{(\zeta-2) / 2}
$$


where

$$
\zeta=\frac{1}{k} \sum_{i=1}^{k} 2\left(\gamma_{i}+1\right)>2, \gamma_{i}=\min \left\{\sigma_{i}, \tau_{i}\right\}
$$

As seen in the proof of Theorem 2, if $u=\left\{u_{m n}\right\}$ is an eventually positive solution of (1), then the lower limit $w$ defined by (10) will satisfy $w>2$ and

$$
\sum_{i=1}^{k} \frac{p_{i} w^{2 \gamma_{i}+2}}{w^{2}-4} \leq 1
$$

By mans of the arithmetic-geometric inequality, we then see that

$$
\begin{aligned}
1 & \geq k\left(\prod_{i=1}^{k} p_{i}\right)^{1 / k} \frac{w^{\zeta}}{w^{2}-4} \geq k\left(\prod_{i=1}^{k} p_{i}\right)^{1 / k} \min _{w>2} \frac{w^{\zeta}}{w^{2}-4} \\
& =k\left(\prod_{i=1}^{k} p_{i}\right)^{1 / k} \frac{\zeta}{8}\left(\frac{\zeta}{\zeta-2}\right)^{(\zeta-2) / 2} 2^{\zeta}
\end{aligned}
$$

as required.

As an example, let us consider the following equation

$$
\left(u_{m+1, n}+u_{m, n+1}\right)^{2}-u_{m n}^{2}+\frac{m^{2}-1}{m^{2}} u_{m, n-1}^{2}=0
$$

Here, the right hand side of the inequality condition in Theorem 3 is equal to $1 / 16$, and the left hand side is 1 . Thus (13) cannot have an eventually positive solution. Note that $\left\{(-1)^{n} m\right\}$ is an oscillating solution of (13).

\section{Dual Equation}

Consider the following equation

$$
\left(u_{m-1, n}+u_{m, n-1}\right)^{2}-u_{m n}^{2}+\sum_{i=1}^{k} p_{i}(m, n) u_{m+\sigma_{i}, n+\tau_{i}}^{2}=0
$$

defined for $m, n=0,1,2, \ldots$, where $p_{1}(m, n), \ldots, p_{k}(m, n)$ are positive functions defined for $m, n \geq 0$, and advancements $\sigma_{1}, \ldots, \sigma_{k}, \tau_{1}, \ldots, \tau_{k}$ are nonnegative integers. This equation may be regarded as a dual of the equation (1). Indeed, dual results parallel to those obtained in the last Section hold. We will state these results as follow. Their proofs, however, will not be given.

Lemma 2. An eventually positive solution $u=\left\{u_{i j}\right\}$ of (14) is also eventually increasing with respect to both of its independent variables. 
Theorem 5. Let $\liminf p_{i}(m, n)=p_{i}$ for $1 \leq i \leq k$. Assume that $\sigma_{i}, \tau_{i} \geq 1$ and that $p_{i}>0$ for $1 \leq i \leq k$. If (14) has an eventually positive solution, then

$$
4 \sum_{i=1}^{k} p_{i}+\sum_{i=1}^{k} P_{i} \leq 1
$$

where $P=\lim \sup p_{i}(m, n)$ for $1 \leq i \leq k$.

Theorem 6. Assume that $\max \left\{\sigma_{1}, \ldots, \sigma_{k}, \tau_{1}, \ldots, \tau_{k}\right\} \geq 1$. If (14) has an eventually positive solution, then

$$
\sum_{i=1}^{k} p_{i} \frac{4^{\mu_{i}}\left(\mu_{i}+1\right)^{\mu_{i}+1}}{\mu_{i}^{\mu_{i}}} \leq 1
$$

where $\mu_{i}=\min \left\{\sigma_{i}, \tau_{i}\right\}$ for $1 \leq i \leq k$.

Theorem 7. Suppose $\eta=\min \left\{\sigma_{1}, \ldots, \sigma_{k}, \tau_{1}, \ldots, \tau_{k}\right\} \geq 1$. If (14) has an eventually positive solution, then

$$
\sum_{i=1}^{k} p_{i} \leq\left(\frac{\eta}{4}\right)^{\eta} \frac{1}{(\eta+1)^{\eta+1}}
$$

Theorem 8. Assume that $\max \left\{\sigma_{1}, \ldots, \sigma_{k}, \tau_{1}, \ldots, \tau_{k}\right\} \geq 1$. If (14) has an eventually positive solution, then

$$
k\left(\prod_{i=1}^{k} p_{i}\right)^{1 / k} \leq \frac{8}{\zeta 2^{\zeta}}\left(\frac{\zeta-2}{\zeta}\right)^{(\zeta-2) / 2}
$$

where

$$
\zeta=\frac{1}{k} \sum_{i=1}^{k} 2\left(\mu_{i}+1\right)>2, \mu_{i}=\min \left\{\sigma_{i}, \tau_{i}\right\}, 1 \leq i \leq k
$$

\section{References}

[1] S. S. Cheng, "Lyapunov inequality conditions for differential and difference equations," Fasiculi Mathematici, 23(1991), 25-41.

[2] B. G. Zhang, S. T. Liu and S. S. Cheng, "Oscillation of a class of delay partial difference equations," J. Difference Eq. Appl., 1(1995), 215-226.

[3] B. G. Zhang and S. T. Liu, "Oscillation of partial difference equations," Pan American Math. J., 5(1995), 61-70.

Department of Mathematics, Bingzhou Normal College, Bingzhou, Shandong 256604, P. R. China.

Department of Mathematics, Tsing Hua University, Hsinchu, Taiwan 30043, R.O.C. 